\title{
PROCESSUS, MODÈLES, PRODUTTS - COMMENT DES ÉTUDIANTS POLONAIS TRADUISENT FLAUBERT
}

\begin{abstract}
A bstract. Dąmbska-Prokop Urszula, Processus, modèles, produits - comment des étudiants polonais traduisent Flaubert [Processes, models, products - how Polish students translate Flaubert]. Studia Romanica Posnaniensia, Adam Mickiewič University Press, Poznań, vol. XXX: 2003, pp. 23-30. ISBN 83-232-1270-8. ISSN 0137-2475.
\end{abstract}

The author presents an analysis of translations of fragments of G. Flaubert's Madame Bovary by 19 students, concentrating on types of errors and difficulties cncountered during this exercise. In the next part of the article a certain typology of these problems and an attempt at explanation of their background are proposed.

Comme l'intention des organisateurs du colloque de Bielefeld était de regarder de plus près non pas le résultat du processus du traduire, le texte traduit, mais au contraire, le processus lui-même et surtout des problèmes concernant l'acquisition de compétences de la rédaction du produit final, c'est sous cet aspect que je me suis proposé de regarder une série de tentatives de traduire un texte, savoir l'allocution de M. Lieuvain, tirée de Madame Bovary (Ed. de poche, 1961, pp.174-178). Mon corpus comprend ici au total 19 copies d'étudiants qui ont traduit ce texte et, en plus, la traduction en polonais faite par Aniela Micińska et publiée plusieurs fois (je m'appuie ici sur Pani Bovary de 1957).

Le travail des étudiants consistait d'abord en une lecture et plusieurs relectures du texte-source, c'est-à-dire de l'allocution du conseiller intégrée dans l'intrigue du roman mais découpée de l'ensemble du roman et considérée, pour des besoins didactiques, comme un tout cohérent.

Pour comprendre le texte, la première étape du travail consistait en une analyse, c'est-à-dire d'abord en des observations concernant les aspects pragmatiques du texte-source: le rôle qu'il joue dans le roman, le type discursif qu'il représente, le contexte politique, social et culturel qui le conditionne, la localisation dans le 
temps, ensuite les aspects textuels, à savoir la progression thématique, les faits linguistiques assurant la cohésion et la cohérence, le rôle des protagonistes, etc. On s'est penché sur l'organisation rhétorique (en entendant par la rhétorique, avec Olivier Reboul, «l'union intime du style et de l'argumentation»; Reboul 1994: 227) et sur les procédés stylistiques les plus importants. C'est ainsi qu'ont été mobilisées les compétences linguistique, rhétorique, littéraire, culturelle et encyclopédique des étudiants. Cette première étape devait permettre aux étudiants à se mettre ensuite au travail de traduire.

Ici, mon intention est, après une brève introduction 1., de m'arrêter, 2., sur quelques-uns des problèmes qu'a posés aux étudiants la compréhension du texte; car les traces de ces difficultés sont visibles dans leurs hésitations, et surtout dans leurs produits définitifs, dans les textes qu'ils se sont décidés à présenter comme rédaction finale.

Ensuite, 3., une typologie de ces difficultés et apories sera suivie par 4., quelques éléments de la critique positive des traductions faites par les étudiants et, à la fin, 5., je terminerai par une conclusion.

1. Il faut d'abord rappeler que, considéré comme un texte à part, détaché du reste du roman, ce fragment constitue un véritable chef-d'œuvre, organisé à l'aide des préformés rhétoriques faciles à reconnaître. On identifie donc facilement le projet de Flaubert, son inventio: dans cette allocution appartenant au discours épidictique, il utilise comme arguments quelques procédés typiques qui sont en même temps les figures rhétoriques des plus banales, surtout captatio benevolentiae et ironie; en effet, le conseiller villageois s'adresse aux paysans en louant d'abord, comme il se doit, le roi et l'administration supérieure, en énumérant ensuite les avantages des efforts quotidiens «des ouvriers des campagnes» concourant à rendre leur situation excellente et digne d'envie. On admire ensuite la dispositio, la construction de l'allocution où un exorde à caractère phatique est suivi de fragments descriptifs parsemés de questions rhétoriques et autres procédés argumentatifs servant à capter l'attention de l'assistance. On relève finalement, au niveau de l'elocutio, nombre de figures de mots, de sens, de construction et de pensée, ainsi que de faits de style au service du projet initial. Sans vouloir trop insister, je dois souligner surtout le rôle qu'y joue la répétition, telle la série amplifiante administration supérieure - gouvernement - monarque - notre souverain - ce roi bien aimé; la rencontre, dans une métaphore empreinte d'ironie, des mots construisant des images bizarres: le char de l'Etat et les périls incessants d'une mer orageuse; ou bien une énumération à caractère ludique races chevalines, bovines, ovines et porcines. Ce qui saute aux yeux, c'est évidemment l'organisation de l'ensemble et le rôle que remplissent, pour assurer la cohérence, les mots de liaison et aussi des éléments textuels, thématiques et «communicationnels»; je mets ce mot entre guillemets, parce que la communication est ici unidirectionnelle: seul parle le conseiller, tandis que le destinataire, les paysans, restent muets bien que présents, évoqués par certains mots, tels messieurs ou vous. Faut-il rappeler également que, 


\title{
STUDIA ROMANICA POSNANIENSIA
}

URSZULA DAMBSKA-PROKOP

Université Jagellonne

\section{PROCESSUS, MODÈLES, PRODUITS - COMMENT DES ÉTUDIANTS POLONAIS TRADUISENT FLAUBERT}

\begin{abstract}
A bstract . Dąmbska-Prokop Urszula, Processus, modèles, produits - comment des étudiants polonais traduisent Flaubert [Processes, models, products - how Polish students translate Flaubert]. Studia Romanica Posnaniensia, Adam Mickiewicz University Press, Poznań, vol. XXX: 2003, pp. 23-30. ISBN 83-2.32-1270-8. ISSN 0137-2475.
\end{abstract}

The author presents an analysis of translations of fragments of G. Flaubert's Madame Bovary by 19 students, concentrating on types of errors and difficulties cncountered during this exercisc. In the next part of the article a certain typology of these problems and an attempt at explanation of their background are proposed.

Comme l'intention des organisateurs du colloque de Bielefeld était de regarder de plus près non pas le résultat du processus du traduire, le texte traduit, mais au contraire, le processus lui-même et surtout des problèmes concernant l'acquisition de compétences de la rédaction du produit final, c'est sous cet aspect que je me suis proposé de regarder une série de tentatives de traduire un texte, savoir l'allocution de M. Lieuvain, tirée de Madame Bovary (Ed. de poche, 1961, pp.174-178). Mon corpus comprend ici au total 19 copies d'étudiants qui ont traduit ce texte et, en plus, la traduction en polonais faite par Aniela Micińska et publiée plusieurs fois (je m'appuie ici sur Pani Bovary de 1957).

Le travail des étudiants consistait d'abord en une lecture et plusieurs relectures du texte-source, c'est-à-dire de l'allocution du conseiller intégrée dans l'intrigue du roman mais découpée de l'ensemble du roman et considérée, pour des besoins didactiques, comme un tout cohérent.

Pour comprendre le texte, la première étape du travail consistait en une analyse, c'est-à-dire d'abord en des observations concernant les aspects pragmatiques du texte-source: le rôle qu'il joue dans le roman, le type discursif qu'il représente, le contexte politique, social et culturel qui le conditionne, la localisation dans le 
temps, ensuite les aspects textuels, à savoir la progression thématique, les faits linguistiques assurant la cohésion et la cohérence, le rôle des protagonistes, etc. On s'est penché sur l'organisation rhétorique (en entendant par la rhétorique, avec Olivier Reboul, «l'union intime du style et de l'argumentation»; Reboul 1994: 227) et sur les procédés stylistiques les plus importants. C'est ainsi qu'ont été mobilisées les compétences linguistique, rhétorique, littéraire, culturelle et encyclopédique des étudiants. Cette première étape devait permettre aux étudiants à se mettre ensuite au travail de traduire.

Ici, mon intention est, après une brève introduction 1., de m'arrêter, 2., sur quelques-uns des problèmes qu'a posés aux étudiants la compréhension du texte; car les traces de ces difficultés sont visibles dans leurs hésitations, et surtout dans leurs produits définitifs, dans les textes qu'ils se sont décidés à présenter comme rédaction finale.

Ensuite, 3., une typologie de ces difficultés et apories sera suivie par 4., quelques éléments de la critique positive des traductions faites par les étudiants et, à la fin, 5., je terminerai par une conclusion.

1. Il faut d'abord rappeler que, considéré comme un texte à part, détaché du reste du roman, ce fragment constitue un véritable chef-d'œuvre, organisé à l'aide des préformés rhétoriques faciles à reconnaître. On identifie donc facilement le projet de Flaubert, son inventio: dans cette allocution appartenant au discours épidictique, il utilise comme arguments quelques procédés typiques qui sont en même temps les figures thétoriques des plus banales, surtout captatio benevolentiae et ironie; en effet, le conseiller villageois s'adresse aux paysans en louant d'abord, comme il se doit, le roi et l'administration supérieure, en énumérant ensuite les avantages des efforts quotidiens «des ouvriers des campagnes» concourant à rendre leur situation excellente et digne d'envie. On admire ensuite la dispositio, la construction de l'allocution où un exorde à caractère phatique est suivi de fragments descriptifs parsemés de questions rhétoriques et autres procédés argumentatifs servant à capter l'attention de l'assistance. On relève finalement, au niveau de l'elocutio, nombre de figures de mots, de sens, de construction et de pensée, ainsi que de faits de style au service du projet initial. Sans vouloir trop insister, je dois souligner surtout le rôle qu'y joue la répétition, telle la série amplifiante administration supérieure - gouvernement - monarque - notre souverain - ce roi bien aimé; la rencontre, dans une métaphore empreinte d'ironie, des mots construisant des images bizarres: le char de l'Etat et les périls incessants d'une mer orageuse; ou bien une énumération à caractère ludique races chevalines, bovines, ovines et porcines. Ce qui saute aux yeux, c'est évidemment l'organisation de l'ensemble et le rôle que remplissent, pour assurer la cohérence, les mots de liaison et aussi des éléments textuels, thématiques et «communicationnels»; je mets ce mot entre guillemets, parce que la communication est ici unidirectionnelle: seul parle le conseiller, tandis que le destinataire, les paysans, restent muets bien que présents, évoqués par certains mots, tels messieurs ou vous. Faut-il rappeler également que, 
prononcée pendant les Comices agricoles, l'allocution d'un haut représentant de l'administration locale est, dans le roman de Flaubert, un élément important servant à promouvoir l'intrigue mais aussi à ridiculiser méchamment tous les protagonistes, leur endimanchement, leur bêtise et platitude? Flaubert bâtit donc cette allocution à l'aide des lieux communs portés à un degré supérieur d'hyperbole et d'ironie et nantit $M$. Lieuvain d'une compétence rhétorique et discursive digne d'admiration. En effet, M. Lieuvain - qui au fond n'a rien à dire (on dirait, avec le perroquet: «tu causes, tu causes, c'est tout ce que tu sais faire») - manipule savamment le public pour provoquer son respect et son enchantement, en s'adressant directement à la foule rassemblée et aussi en impressionnant l'assistance par une disposition habile des accents forts, un rythme expressif, emploi adroit des connecteurs liant différentes parties, etc., etc.

2. Sans insister davantage sur les particularités du texte-source, je passe aux travaux des étudiants où se vont voir immédiatement des difficultés manifestes qu'ils ont rencontrées au cours de leur travail.

a. Une des premières questions d'ordre discursif qu'ils se sont posées était de savoir si la formule d'adresse, messieurs, doit, oui ou non, passer intacte, c'est-àdire si le mot polonais 'panowie' suffit dans une traduction faite au $\mathrm{XX}^{\mathrm{e}}$ ou au XXI' siècle, traduction destinée à un lecteur contemporain; c'est en tout ainsi, comme notre contemporain, qu'ils ons identifié le récepteur, ou le commanditaire, de ces traductions. Réponse: sur 19 copies d'étudiants et une traduction publiée, on a proposé 18 fois de garder le mot 'panowie', l'auteur de (5) a choisi la formule 'proszę państwa', celui de (10) - 'szanowni państwo'. Commentaire: ces deux étudiants se sont peut-être laissé impressionner, ou suggérer, par une question que j'ai leur avais posée exprès, voulant attirer leur attention sur le rôle des pronoms et des formules d'adresse utilisés dans ce texte, notamment si l'emploi du seul messieurs ne serait aujourd'hui choquant, vu que le public se composait aussi bien d'hommes que de femmes.

b. Il est significatif d'observer chez tous les étudiants une tendance à éviter les pronoms: par exemple, à 7 vous présents dans les 4 premiers alinéas correspondent, dans la plupart des traductions, 3 fois 'wy' là où le texte français emploie la forme forte (emphatique) du pronom; ailleurs plusieurs solutions ont été adoptées pour éviter le pronom. Ce fait s'explique dans l'optique comparative, parce que, en polonais, une désinence du verbe conjugué suffit à elle seule à exprimer la personne et le nombre. Ce fait s'explique également, semble-t-il, par un incomfort ressenti par les étudiants cherchant à contoumer en polonais le problème, c'est-à-dire à éviter la lourdeur de la forme 'podzielicie', 'podzielacie' ou 'zgodzicie się ze mną'. C'est ainsi, par exemple, que la phrase et ce sentiment... sera partagé par vous tous est rendue par la traductrice "officielle» par une phrase équivalente 'a pragnienie to... podzielicie ze mną wszyscy'; chez les étudiants, on observe différentes solutions:

(4): 'będzie to i wasze odczucie';

(5): 'wszyscy panowie podzielą uczucia'; 
(6): 'wszyscy podzielą moje odczucie';

(8): 'odczucie to...podzielą wszyscy tutaj zebrani';

(13): 'to uczucie... wszyscy podziela';

(15): 'to uczucie... zostanie przez wszystkich podzielone';

(18): 'uczucie to stanie się udziałem was wszystkich';

et enfin (20) - 'ten nastrój udzieli się każdemu z nas'.

Il est donc à remarquer que dans ces versions, à l'exception de (15), la forme passive a été transformée en forme active, ce qui paraît conforme au «génie» de la langue polonaise. Ce qui frappe, c'est une tendance à remplacer la phrase à forme verbale équivalente 'podzielicie' (= vous partagerez) par une autre construction, avec un autre verbe, comme dans ( 4 - être), ( 18 - devenir) et ( 20 - passer), ou bien à mettre en fonction de sujet le pronom indéfini 'wszyscy' (tous) ou, dans (20) 'każdy' (chacun). Il est clair que la forme équivalente, la deuxième personne du pluriel, a été ici refusée par les étudiants pour des raisons esthétiques: leur effort à s'exprimer d'une façon moins rigide pour l'oreille, donc considérée comme meilleure, est ici sensible.

c. Un autre problème est posé par la traduction de l'infinitif. Il apparaît 5 fois dans les deux premiers fragments, tandis que, chez les étudiants, son emploi se réduit à 2 cas, rarement à 3 cas, à 1 cas, ou même aucun. C'est ainsi qu'on traduit l'infinitif modalisé (qu'il me soit permis... de rendre justice à l'administration...; et aussi sachant... faire respecter la paix...), tandis que dans d'autres cas l'infinitif (dans avant de vous entretenir et dans tremblaient de se voir révéillés) est rendu en polonais par un substantif déverbal ou par un nom simple. Ce fait est intéressant dans une optique comparative de même que dans une optique sémantique, puisqu'il s'agit des connotations intersubjectives de lourdeur qu'évoquerait en polonais un enchaînement d'infinitifs. Pour éviter ce type de maladresse, les étudiants cherchent plutôt différents synonymes et remplacent l'infinitif par des substituts: surtout substantif déverbal ou proposition complétive à verbe funi. De même, au lieu d'entasser une suite de substantifs déverbaux - 'omówienie', 'spotkanie', 'pragnienie' dans (1), ils préfèrent recourir à des formes verbales comme p.ex. 'wyjawię przedmiot' et 'mocno wierzę' dans (4); ailleurs ils emploient des noms simples, tels 'temat' ou 'odczucie', 'pogląd' i 'szacunek', ou des syntagmes nominaux, tel 'przedmiot dzisiejszego spotkania', etc., etc.

d. Il faudrait s'arrêter bien sûr aussi sur les problèmes lexicaux, sur le choix des mots qui, dans les copies des étudiants, attirent attention. Une lecture à même le texte de la phrase ce roi bien-aimé...qui dirige à la fois d'une main si ferme et si sage le char de l'Etat parmi les périls incessants d'une mer orageuse a abouti chez quelques-uns des étudiants à une traduction mot-à-mot, p.ex. "który dłonią zarazem tak zdecydowaną i tak zręczną kieruje zaprzęgiem Państwa pośród nieustannych niebezpieczeństw wzburzonego morza' (3); à côté de 'zaprzęg' je relève les mots 'rydwan' ( 8 fois), les deux mots appartenant au registre élevé; 'powóz' et 'wóz'; 'ster', 'okręt' et 'nawa'; dans une copie il y a même 'nasz kraj', donc la métaphore 
a été ici rejetée et son sens a été interprété. A l'occasion, il s'est avéré qu'un nombre d'étudiants ne savaient du mot 'nawa' que son acception architecturale: la nef d'une église, la connaissance du latin laissant à désirer.

e. Ce qui semble donc surtout intéressant, ce sont les modèles, ou comme dirait Antoine Berman, les projets de traduire adoptés par les étudiants. Ces projets montrent des hésitations, visibles à travers les inconséquences, souvent même une compétence insuffisante de la langue maternelle, ou parfois l'incompréhension du sens du texte-source.

Observons les traductions des étudiants sous ce demier point. La phrase sachant d'ailleurs faire respecter la paix comme la guerre, l'industrie, le commerce, l'agriculture et les beaux arts est certes difficile à rendre en polonais qui n'a pas de construction factitive; d'autre part, certains étudiants n'ont pas saisi l'ironie de l'énumération amplifiante... Si donc la traductrice du roman propose une équivalence approximative, les étudiants cherchent des solutions différentes. Certains, comme la traductrice (1), réussissent des tournures équivalentes (2), (8), (10), (11), (12) - mais d'autres n'ont pas compris la construction et tantôt attribuent à la construction faire respecter le sens actif (4) ou (9), tantôt en modifient le sens: si (5) paraît encore admissible, (6) change complètement le sens et, de plus, l'étudiant a senti le besoin d'introduire un verbe complémentaire:

(1): 'umiejacc przy tym nakazać poszanowanie tak dla wojny, etc.'

(2): 'umiejąc jednocześnie nakazać poszanowanie',

(8): 'potrafiąc nauczyć szacunku zarówno dla pokoju, etc.',

(10) et (11): 'umiejąc ponadto wzbudzić szacunek/poszanowanie',

(7) se sert d'une paraphrase: 'za którego sprawą panuje poszanowanie tak wojny, jak i pokoju...'

de même (12): 'potrafiąc sprawic, by szanowany był pokój...'.

(4): 'umiejąc równo cenić czas wojny, etc.',

(9): 'który jednako umie docenić...';

(5): 'który umie nauczyć poważania dla pokoju, etc.',

(6): 'potrafiąc narzucić wojnę jak i pokój, zadbać o przemysł, etc.'

f. On pourrait aussi accuser les étudiants de n'avoir pas compris le texte dans d'autres fragments. Si, au deuxième alinéa, la traductrice a changé la perspective fonctionnelle de la phrase, en offrant une solution qu'on peut considérer comme heureuse (1) - les étudiants ont eu visiblement des difficultés tant au niveau syntaxique, que surtout en ce qui concerne le choix d'un registre, des mots utilisés, mais aussi du «ton» de ce fragment, p.ex.:

(1): 'Minął już czas, panowie, gdy wśród waśni domowych spływały krwią place naszych miast, gdy obywatel, kupiec, robotnik nawet, układając się wieczorem do spokojnego snu, drżał na myśl, że zbudzą go nagle dzwony na pożar'. 
(3): 'Minęły czasy, panowie, kiedy niezgoda społeczna topiła we krwi nasze place, kiedy posiadacz ziemski, handlarz, a nawet robotnik, zasypiając wieczorem snem sprawiedliwego, drżeli na myśl, że nagle zbudzeni zostaną odgłosem wezwania do pożaru'.

On réflechit sur les motifs du choix des mots 'posiadacz ziemski' ou 'handlarz' et des connotations qu'ils véhiculent; sur l'emploi de la locution toute faite 'snem sprawiedliwego' à la place de d'un sommeil paisible; sur le groupe 'odgłosem wezwania do pożaru' remplaçant le bruit des tocsins incendiaires et donnant à cette expression un aspect quelque peu ridicule. Que dire si, dans (18), cette expression est remplacée par 'buntownicza wrzawa'?

Que dire aussi à propos des textes suivants, (20) et (19), temoignant d'une incompréhension?

(19): 'Skończył się czas, Panowie, gdy obywatelska niezgoda spływała krwią na naszych placach publicznych, gdy właściciel, negocjator, sam robotnik, zasypiając wieczorem spokojnym snem, drżeli na myśl o nagłej pobudce przy dzwonach alarmujących pożar',

(20): 'Minęły już czasy, Panowie, kiedy niezgoda obywatelska plamiła krwią miejskie place, kiedy ziemianin, kupiec, robotnik nawet, zasypiajac noca spokojnym snem, drżeli, obudzeni nagle biciem dzwonów ogłaszających pożoge'?

3. Est-ce donc c'est ce texte particulier qui est hérissé tout particulièrement de difficultés quasi insurmontables à tel point qu'il est impossible aux étudiants polonais de donner du texte-source une "copie» relativement conforme? Ou bien ces obstacles et difficultés sont à chercher ailleurs?

Il est trop facile de s'arrêter sur des fautes, commises par rapport aux normes linguistiques - syntaxiques, lexicales, sémantiques, euphoniques. Il l'est moins d'accuser les infractions aux règles rhétoriques, textuelles, discursives, et aussi idéologiques (si l'on pense à la grille d'analyse textuelle proposée par Lita Lundquist). Car le texte de Flaubert demande pour être correctement traduit de prendre en considération nombre de facteurs, et parmi cux, peut-être en premier lieu, une compétence encyclopédique. Pourquoi Flaubert parle-t-il de la religion, plus affermie (après les activités du Parti de l'Ordre), que sont les grands centres manufacturiers, expression que certains traducteurs ont rendue par 'wielkie centra rękodzielnicze', ou bien 'manufakturowe' - mais par exemple (3) et (6) ont déjà 'wielkie ośrodki fabryczne', sachant peut-être qu'en France sous le Second Empire fleurissait déjà l'industrie moderne, bien que Flaubert, en situant l'action du roman quelques années plus tôt, sous le règne de Louis-Philippe, puisse encorc parler des manufactures. Les traducteurs devraient aussi disposer des compétences textuelles. identifier le type de texte et les conséquences qui en découlent, déchiffrer le sens caché et savoir lire entre les lignes, être sensibles aux collocations des mots ou 
clichés dont se sert le romancier qui raille méchamment, sans pitié, le bourgeois et le monde qui l'entoure.

4. Pourrait-il en être autrement? - Je passe en ce moment au point 4. de mon exposé et rappelle: Selon Antoine Berman, la critique positive des traductions s'appuie sur quelques pilliers. Elle commence, dit-il, par les «lectures de la traduction, puis, bien séparées de celles-ci, celles de l'original. La dialectique propre à ces lectures me mène à l'auteur du texte traduit» pour savoir «qui est le traducteur», quelle est sa position traductive, son projet de traduction et son horizon traductif». Ainsi préparée, la critique "productive» de Berman sert, selon lui, à «éclairer le pourquoi de l'échec traductif» d'un côté, et d'autre part à «préparer l'espace de jeu d'une retraduction sans faire le 'donneur de conseils'» (1995: 17-18).

Il serait donc intéressant d'indiquer des éléments des modèles que les étudiants ont choisis et appliqués dans leurs traductions. Il faut encore une fois s'arrêter d'abord sur les aspects négatifs, parce que leur manque d'expérience est évident et d'ailleurs attendu. Ce qui donc frappe dans leurs projets du traduire, c'est aussi bien l'aspect linguistique que l'aspect textuel: leurs hésitations à adopter d'une façon conséquente un registre unique (p.ex. mélange du type de 'Szanowni Państwo' à côté de 'wy, rolnicy i parobcy wiejscy' dans [10]; colloquialismes, p.ex. 'nie te czasy' dans [14]; constructions syntaxiques maladroites ou même incorrectes, p.ex. 'drżeli $z$ obawy bycia nagle zbudzonym hałasem bicia na alarm przeciwpożarowy' dans le même texte [14]; ou enfin quelques fautes de compréhension, p.ex. 'buntownicza wrzawa' à la place des tocsin incendiaires toujours dans [14]). A côté du manque d'expérience - il est donc évident que certains étudiants ont aussi des lacunes dans la compétence de leur langue maternelle. Mais si l'on réflechit sur le «pourquoi» de l'échec de leurs tentatives, on revient à l'idée exprimée par Berman disant qu'il appartient au critique également de "préparer l'espace de jeu d'une retraduction» et de constituer ainsi ce qu'il avait appelé «la translation d'une (2uvre». Les traductions des étudiants, tout défectueuses qu'elles sont quelquefois, sont utiles parce qu'elles permettent, je l'espère, la translation dont parle Berman, c'est-à-dire l'entrée dans la langue-culture polonaise de textes qui pourraient, après correction, être considérés comme «de nouveaux originaux», constituant une série (au sens de Balcerzan) et déterminés par des conditions pragmatiques changées, changées par rapport au texte-source, changées aussi par rapport à l'unique traduction publiée jusqu' aujourd'hui.

5. En guise de conclusion, je voudrais insister sur le fait que les compétences traductologiques, y comprises les compétences rédactionnelles, s'acquièrent lentement, laborieusement, à petits pas. Certes, il importe de mettre l'accent sur les TAPs (tkink-aloud protocols) permettant d'avoir accès à ce qui se passe dans la «boite noire» du traducteur. Il faut insister aussi sur l'importance de l'analyse dite procédurale et autres nouvelles techniques rédactionnelles. Mais je ne peux pas 
perdre de vue que le processus du traduire est fonction. de toutes les compétences mentionnées plus haut: si l'étudiant n'en dispose pas, si son projet n'est pas assis sur une base satisfaisante, son produit échoue. D'autre part, même s'il échoue en tant que "mauvaise copie» de l'original - il peut intéresser la critique des traductions en tant qu'étape menant à la «translation».

\section{BIBLIOGRAPHIE}

B erman, A. (1995), Pour une critique des traductions, Johm Donne, Paris, Gallimard. Lundquist, L. (1983), L'analyse textuelle, Paris, CEDIC.

Reboul, O. (2e éd. 1994), Introduction à la rhétorique, Paris, PUF. 